\title{
Tipifarnib, a potential adjuvant that unlocks therapeutic resistance by normalizing microenvironment
}

\author{
Lin Tian ${ }^{1 *}$ and Sicong Zhang $^{2}$ \\ ${ }^{1}$ Cancer Biology and Genetics Program, Sloan Kettering Institute, Memorial Sloan Kettering Cancer Center, 1275 York Avenue, New York, NY 10065, USA \\ ${ }^{2}$ Laboratory of Biochemistry and Molecular Biology, The Rockefeller University, 1230 York Avenue, New York, NY 10065, USA
}

\section{Introduction}

More than 1.5 million new cancer cases are diagnosed in US every year [1]. Unlike the normal cells that cooperate with each other to form an ecosystem within the tissue, the cancer cells break the rules by bypassing the normal proliferation, breaching the basement membrane, disseminating into circulation and finally establishing distant colonization [2]. To initiate this malignant cellular program, cancer cells hijack a variety of existing cellular mechanisms that are critical to normal tissue development and regeneration [3]. Despite the increasing knowledge on these molecular regulators leveraged by cancer cells to fulfill the aberrancy, most advanced cancers are still incurable, indicating the therapies to treat cancer have not kept up. In fact, the majority of current therapeutic regimens are designed to target cancer cells. However, the neighbors of cancer cells - especially the connective tissue or stroma - are far from passive bystanders [4]. Tumor-associated stromal cells including endothelial cells, fibroblasts and immune cells can also behave aberrantly in tumor microenvironment and contribute to disease progression and therapeutic resistance [2]. An important hallmark of cancer is the dysregulation of angiogenesis, which leads to abnormal vascular network within the tumor that is filled by dilated, tortuous, and hyperpermeable vessels [5]. It has been reported that normalization of tumor vasculature by inhibiting the pro-angiogenic activity of vascular endothelial growth factor (VEGF) can improve the efficacy of chemotherapy and immune checkpoint blockade therapy [68]. In this short communication, we extend the concept of "vascular normalization" to "microenvironment normalization". The underlying hypothesis is that alteration of cancer-associated stroma abates the drug delivery and therefore limits the efficacy of therapeutic regimen. Thus, normalizing tumor-associated stromal components through molecular or pharmacologic perturbation might provide a novel opportunity for cancer treatment by enhancing the efficacy of conventional therapy and immune infiltration, in addition to antiangiogenesis therapy (Figure 1).

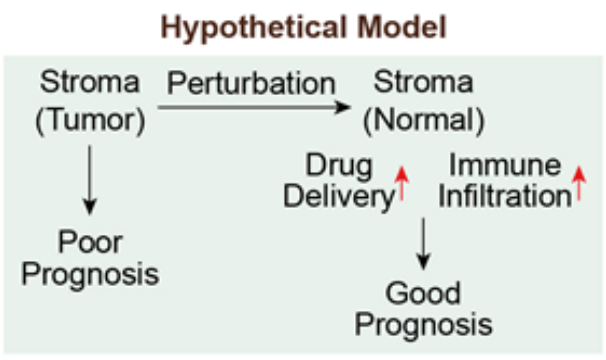

Figure 1. Microenvironment normalization hypothetical model. Hypothetical model describing that normalizing microenvironment can increase the efficacy of therapeutic intervention and improve prognosis outcome.

\section{Results}

We first ask which cancer type can be the best model to test microenvironment normalization hypothesis. We think the ideal model should have high abundance of stroma components. To estimate the percent of stromal cells within the tumor, we used ESTIMATOR [9], a computational tool that uses gene expression data as input and predicts the tumor purity, to estimate the abundance of stromal cells. Across 22 different tumor types in TCGA database, we found that the pancreatic tumors are of the highest stromal scores (Figure 2a), which is consistent with previous studies [10]. Importantly, the high stroma scores correlate with poor prognosis in pancreatic cancer patients (Figure $2 \mathrm{~b}$ ). It is also notable that the progression of pancreatic cancer is accompanied by the increase of stroma, indicating that the stromal cells within malignant tissues may have evolved to cope with tumor progression (Figure 2c). Based on these observations, "microenvironment normalization" may bring the great therapeutic benefit for pancreatic cancer patients.

We next investigate which stroma-related pathways are enriched in pancreatic adenocarcinoma tissues versus normal tissues. We found the hydrolase activity is substantially increased in pancreatic adenocarcinoma (Figure $3 \mathrm{a}, \mathrm{b}$ ). Indeed, in pancreatic cancers, the serine hydrolase RBBP9 has been found to promote anchorageindependent growth in vitro and tumor-initiation in vivo [11]. In addition, hyaluronidase inhibitor PEGPH20 has been developed to reduce the "desmoplastic reaction" in the stage IV metastatic pancreatic cancer and is currently in the Phase II clinical trial. However, since hydrolase activity is also essential to diverse cellular processes such as myofibroblast and fetal development, systemic inhibition of hydrolase activity can cause side effect on other healthy tissues. Therefore, we queried the L1000CDS ${ }^{2}$ database to search for other compounds that can adverse the hydrolase activity (Figure 4a). L1000CDS ${ }^{2}$ is a dataset that integrates the LINCS L1000 small molecule expression profiles and is under development by Maayan Lab at the Icahn School of Medicine at Mount Sinai [12]. The L1000CDS ${ }^{2}$ dataset will search for the small molecules that can potentially reverse the custom defined gene signature. We found that the top candidate compounds target the Ras signaling (Figure $4 \mathrm{~b}$ ). Indeed, more than $50 \%$ of the pancreatic tumors carry Ras mutation (Figure 4c). And inhibiting Ras signaling in cancer cells alone may be an effective strategy to treat pancreatic cancers. Based

${ }^{\star}$ Correspondence to: Lin Tian, Cancer Biology and Genetics Program, Sloan Kettering Institute, Memorial Sloan Kettering Cancer Center, 1275 York Avenue, New York, NY 10065, USA, E-mail: lintian0616@gmail.com

Received: May 14, 2018; Accepted: May 27, 2018; Published: May 30, 2018 
a
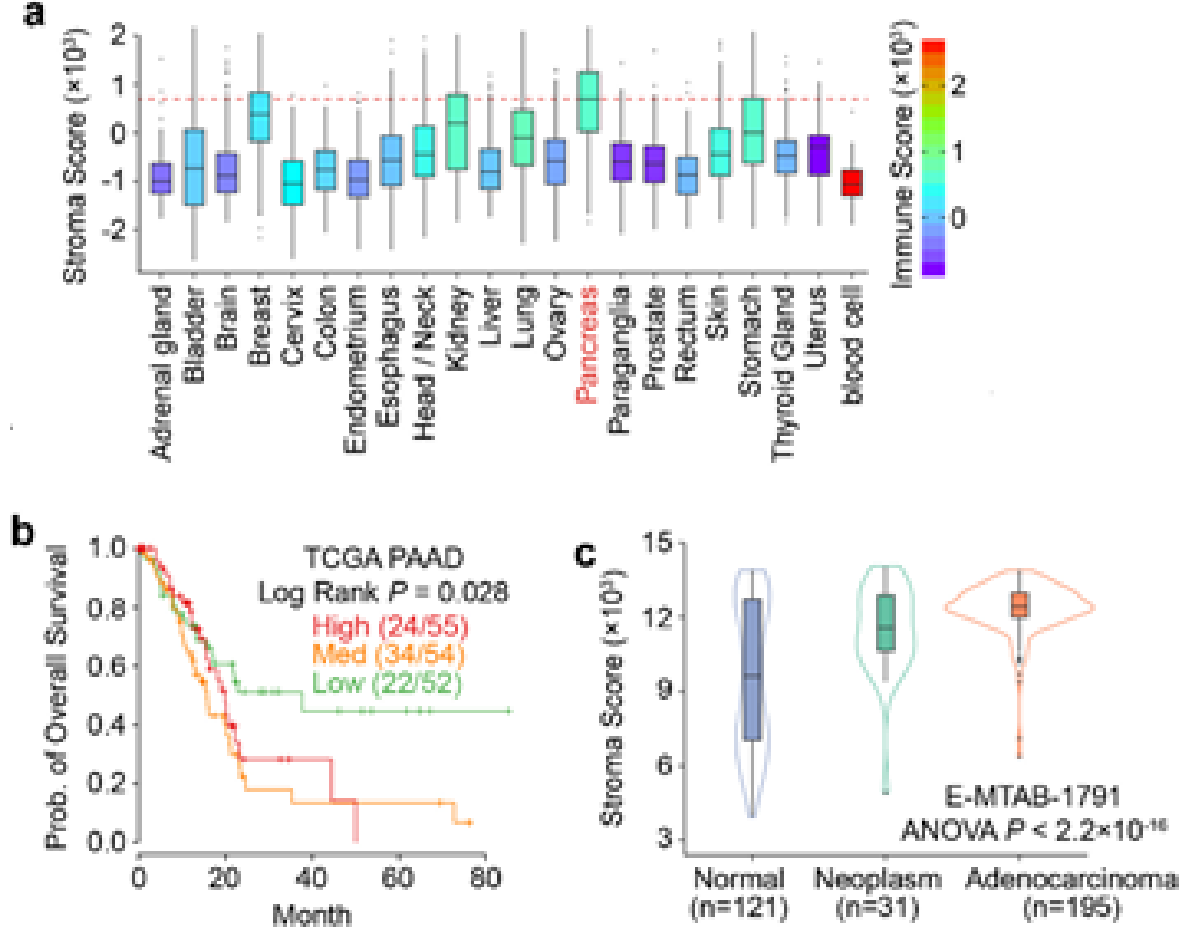

Figure 2. Pancreatic cancer as the model to test microenvironment normalization hypothesis. a) Comparison of stroma and immune scores across 22 tumor types in TCGA dataset. The box and whisker plots showing the abundance of stromal cells, and the color key representing the percent of immune cells within the tumor. b) Kaplan-Meier curves showing that high stromal abundance in pancreatic tumors is associated with poor survival outcome. c) Comparison of stroma scores among normal, neoplasm and adenocarcinoma tissues in pancreas.

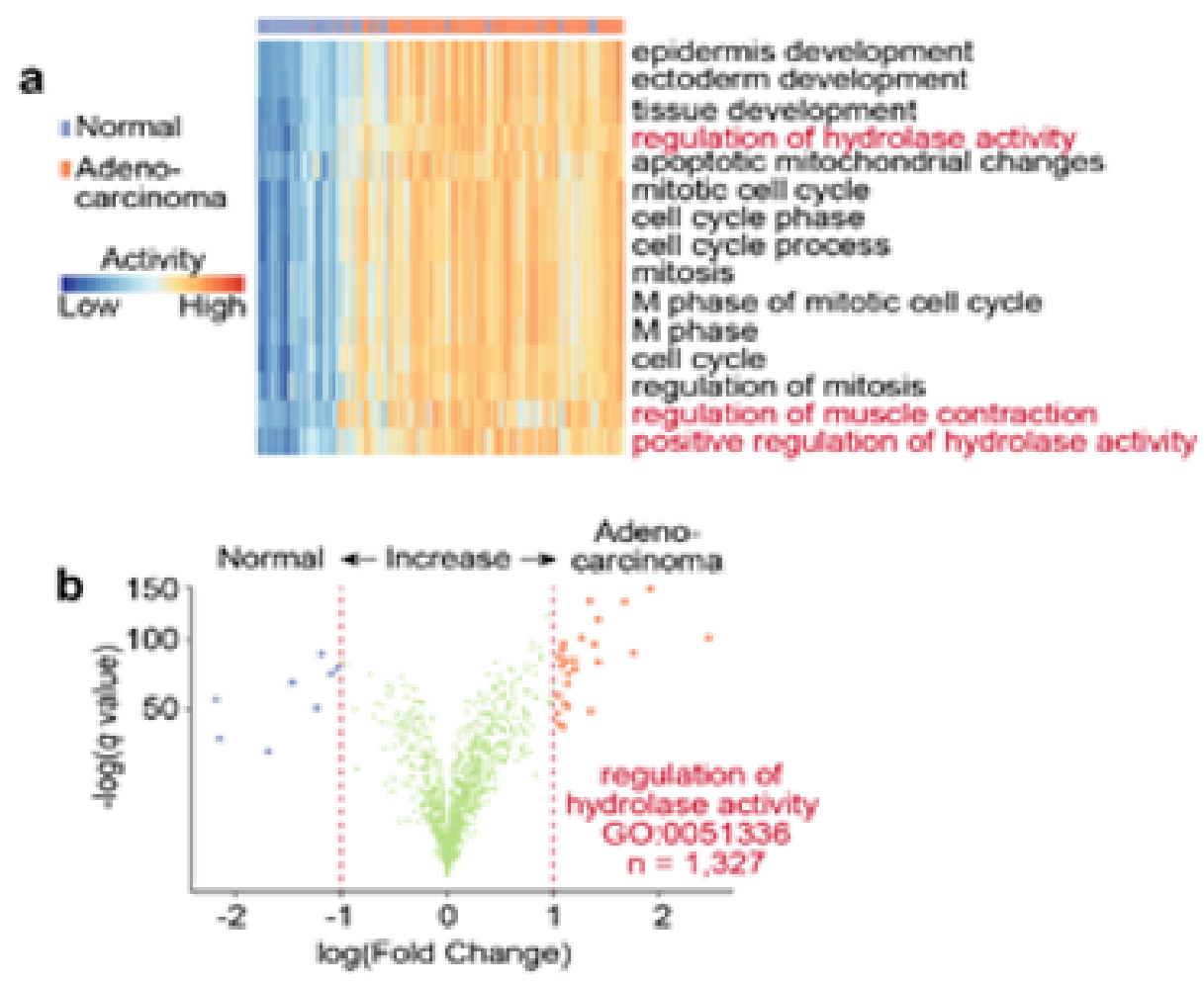

Figure 3. Pancreatic Cancer Progression is Accompanied by the Increased Hydrolase Activity. a) Heat map showing the top 15 gene signatures enriched in adenocarcinoma versus normal pancreatic tissues. Column-side annotation shows the types of the samples. b) Volcano plot showing the differential expression of the genes ( $\mathrm{n}=1,327)$ in Regulation of Hydrolase Activity (GO:0051336). The expression difference between adenocarcinoma and normal tissues is plotted on the $x$ axis (log scale), and the false discovery rate (adjusted significance) is plotted on the $y$ axis (-log scale). Upregulated and downregulated genes in adenocarcinoma are labeled with red and blue, respectively. 
a

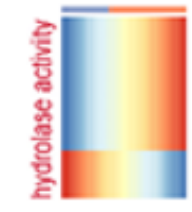

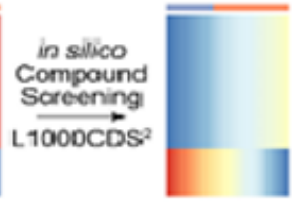

b

\begin{tabular}{ccccc}
$\alpha$ & Compound & Target & Cell & Dosage \\
\hline $141^{\circ}$ & PD184352 & MEK & MCF10A & $1.11 \mu \mathrm{M}$ \\
$139^{\circ}$ & Tipifarnib & Ras & OV7 & $10 \mu \mathrm{M}$ \\
$138^{\circ}$ & JSH-23 & NF-kB & MDST8 & 64M \\
$138^{\circ}$ & KINO01-055 & JAK3 & A549 & $10 \mu \mathrm{M}$ \\
$138^{\circ}$ & R3904 & Aurora & PHH & $10 \mu \mathrm{M}$
\end{tabular}

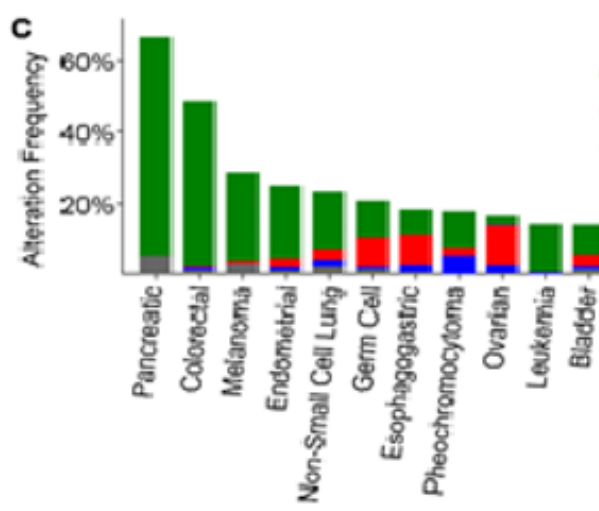

- Mutation Fusion
- Amplification Deep Deletion
- Multiple Alterations.

d

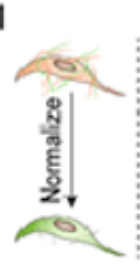

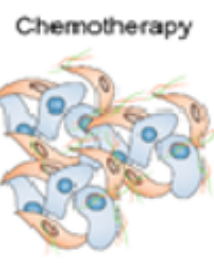
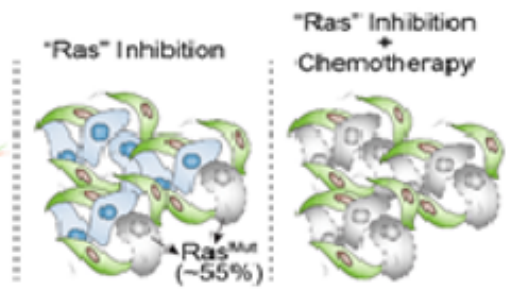

Figure 4. Inhibition of Ras Signaling May Normalize tumor associated microenvironment. a) Schematic diagram showing the principle of in silico chemical screening using L1000CDS database. b) A table showing the top candidate compounds that can adverse the pancreatic adenocarcinoma-specific hydrolase gene signature. The effect of compound is calculated based on the $\alpha$ degree range from $0^{\circ}$ (stimulation) and $180^{\circ}$ (inhibition). c) Bar plot showing the mutation frequency of Ras (K-, H-, N-Ras) mutations across 24 cancer types in TCGA dataset. d) Expected working model: as more than $50 \%$ of the pancreatic tumors carry Ras mutation, the inhibitors targeting RAS signaling mediators including MEK, JAK and Aurora will be effective on malignant cells. In addition, the suppression of Ras signaling in tumor stroma may inhibit the hydrolase activity, thereby normalizing microenvironment and providing promising strategies for potential synergy with chemotherapy.

on our bioinformatics prediction, suppressing Ras signaling may also normalize microenvironment and increase therapeutic efficacies, which may present promising strategies for potential synergy with chemotherapy and immune checkpoint therapy, respectively (Figure $4 \mathrm{~d}$ ).

Tipifarnib [13], a potent and specific farnesyltransferase inhibitor, ranks among the top compounds that can reverse the hydrolase activity in pancreatic cancer (Figure $4 \mathrm{~b}$ ). Upon treatment with Tipifarnib, the prenylation of the CxxX tail motif in Ras kinase will be blocked, which prevents Ras from binding to the membrane upon activation. As a result, Tipifarnib prominently abates the proliferation of Ras mutant cells. However, the activity of membrane-free cytoplasmic Ras is not suppressed by Tipifarnib, indicating Tipifarnib monotherapy may not be effective. This might also partially explain why Tipifarnib was suspended in Phase III clinical trials [14].

\section{Discussion}

Despite the ineffectiveness of Tipifarnib monotherapy, Tipifarnib now provides the opportunity to address the questions associated with the "microenvironment normalization" hypothesis. How does the hydrolase activity change in pancreatic cancer stroma after Tipifarnib treatment? And, how does the treatment of Tipifarnib alter pancreatic cancer microenvironment, such as hypoxia, and drug delivery efficiency? It is also notable that immune checkpoint blockade therapy works poorly for pancreatic cancer, presumably due to the low immune infiltration efficiency. It will be interesting to investigate whether the combination of anti-PD1/anti-CTLA with Tipifarnib can enhance immune cytotoxic and forge the innovative combinatory intervention to pancreatic cancer.

\section{Methods}

TCGA RNA-seq data were download from UCSC Cancer Genome Browser (https://genome-cancer.ucsc.edu/) and used as input for the $\mathrm{R}$ package of ESTIMATOR to estimate the stroma percent. Gene signature were calculated using single sample Gene Set Enrichment Analysis (ssgGSEA) on the server of GenePattern (https://genepattern. broadinstitute.org/gp). In silico drug screening was performing using the online graphical user inference of L1000CDS ${ }^{2}$ (http://amp.pharm. mssm.edu/L1000CDS2)

\section{References}

1. Siegel RL, Miller KD, Jemal A (2018) Cancer statistics. CA Cancer J Clin 68: 7-30. [Crossref]

2. Hanahan D, Weinberg RA (2015) Hallmarks of Cancer: The Next Generation. Cell 144: 646-674. [Crossref]

3. Martin GS (2003) Cell signaling and cancer. Cancer Cell 4: 167-174. [Crossref]

4. Binnewies M, Roberts EW, Kersten K, Chan V, Fearon DF, et al. (2018) Understanding the tumor immune microenvironment (TIME) for effective therapy. Nat Med 24: 541550. [Crossref] 
5. Jain RK (2005) Normalization of tumor vasculature: an emerging concept in antiangiogenic therapy. Science 307: 58-62. [Crossref]

6. Tian L, Goldstein A, Wang H, Ching Lo H, Sun Kim I, et al. (2017) Mutual regulation of tumour vessel normalization and immunostimulatory reprogramming. Nature 544: 250-254. [Crossref]

7. Huang Y, Goel S, Duda DG, Fukumura D, Jain, RK (2013) Vascular normalization as an emerging strategy to enhance cancer immunotherapy. Cancer Res 73: 2943-2948. [Crossref]

8. Huang Y, Yuan J, Righi E, Kamoun WS, Ancukiewicz M, et al. (2012) Vascular normalizing doses of antiangiogenic treatment reprogram the immunosuppressive tumor microenvironment and enhance immunotherapy. Proc Natl Acad Sci U S A 109: 17561-17566. [Crossref]

9. Yoshihara K, Shahmoradgoli M, Martínez E, Vegesna R, Kim H, et al. (2013) Inferring tumour purity and stromal and immune cell admixture from expression data. Nat Commun 4: 2612. [Crossref]
10. Erkan M, Hausmann S, Michalski CW, Fingerle AA, Dobritz M, et al. (2012) The role of stroma in pancreatic cancer: diagnostic and therapeutic implications. Nat Rev Gastroenterol Hepatol 9: 454-467. [Crossref]

11. Shields DJ, Niessen S, Murphy EA, Mielgo A, Desgrosellier JS, et al. (2010) RBBP9: a tumor-associated serine hydrolase activity required for pancreatic neoplasia. Proc Natl Acad Sci U S A 107: 2189-2194. [Crossref]

12. Duan Q, Reid SP, Clark NR, Wang Z, Fernandez NF, et al. (2016) L1000CDS2: LINCS L1000 characteristic direction signatures search engine. Npj Syst Biol Appl 2: 16015. [Crossref]

13. Alsina M, Fonseca R, Wilson EF, Belle AN, Gerbino E, et al. (2004) Farnesyltransferase inhibitor tipifarnib is well tolerated, induces stabilization of disease, and inhibits farnesylation and oncogenic/tumor survival pathways in patients with advanced multiple myeloma. Blood 103: 3271-3277. [Crossref]

14. Van Cutsem E, van de Velde H, Karasek P, Oettle H, Vervenne WL, et al. (2004) Phase III trial of gemcitabine plus tipifarnib compared with gemcitabine plus placebo in advanced pancreatic cancer. J Clin Oncol 22: 1430-1438. [Crossref]

Copyright: $\mathbb{C} 2018$ Tian L. This is an open-access article distributed under the terms of the Creative Commons Attribution License, which permits unrestricted use, distribution, and reproduction in any medium, provided the original author and source are credited. 